\title{
Nocturnal atrial fibrillation in a patient with obstructive sleep apnoea
}

A 75 year old woman was referred to our hospital for suspected sleep apnoea. Her medical history mentioned arterial hypertension and paroxysmal atrial fibrillation (AF). Polysomnography revealed obstructive sleep apnoea (OSA). During one phase of OSA the heart rate, determined by signals derived from pulse oximetry, showed a sharp rise in frequency (fig 1, left). Simultaneously recorded electrocardiography (ECG) indicated AF (fig 2A). The onset of AF was preceded by a long apnoeic event $(48 \mathrm{~s})$ with marked oxygen desaturation $\left(\mathrm{SaO}_{2} 67 \%\right)$. After a period without apnoeas, spontaneous reversal to sinus rhythm occurred (fig 1, right; fig 2B).

OSA has been reported to be associated with an increased risk of recurrence of AF after primarily successful electrical cardioversion, ${ }^{1}$ and sleep disordered breathing has been found to be very common in patients with AF. ${ }^{2}$ The present report gives the first direct evidence that OSA might trigger episodes of AF. Although we cannot definitively exclude the

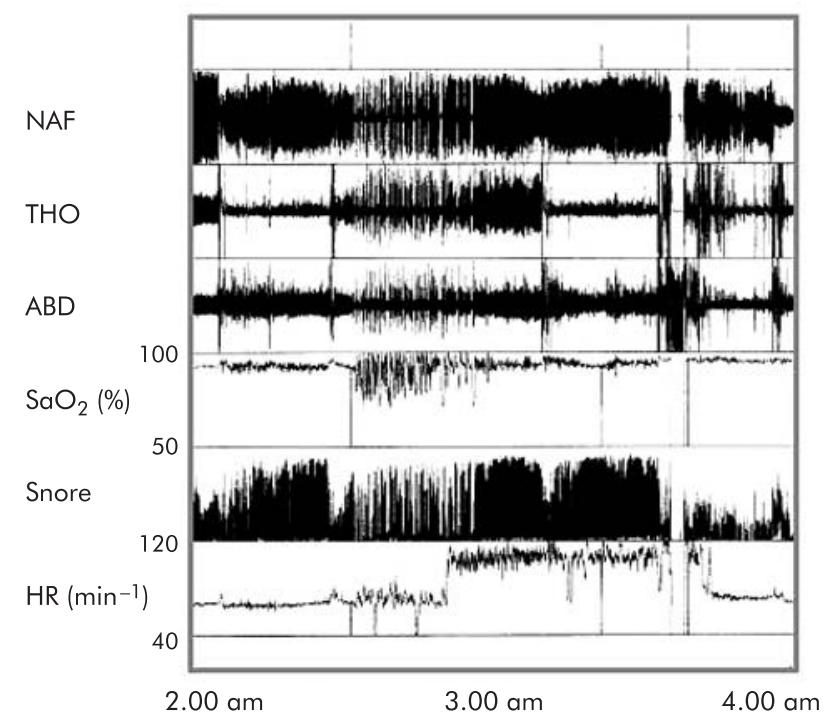

Figure 1 Two hour polysomnographic recording showing onset of AF during a period of apnoeas with consecutive desaturations and spontaneous resolution after a phase of undisturbed breathing. NAF, oronasal flow; $\mathrm{THO} / \mathrm{ABD}$, thoracoabdominal breathing; $\mathrm{SaO}_{2}$, oxygen saturation; $H R$, heart rate.

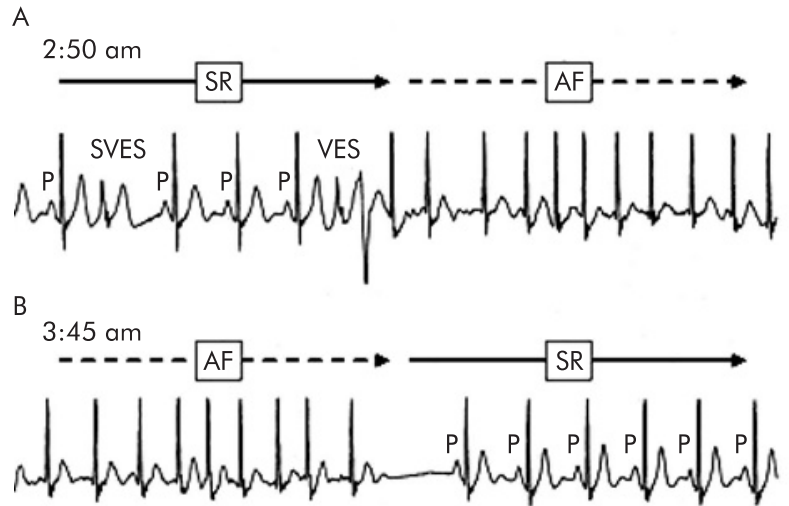

Figure 2 Single channel ECG recordings (lead I, $30 \mathrm{~s}$ intervals) showing $(A)$ times of $A F$ onset and $(B)$ reversal to sinus rhythm. $A F$ is characterised by an irregular pattern of QRS complexes in the absence of $p$ waves. SVES, supraventricular extrasystole; VES, ventricular extrasystole; SR, sinus rhythm.

possibility that our observations are coincidental, we suggest a causal association between OSA and the onset of AF. Since AF occurred immediately after a long lasting apnoea with severe oxygen desaturation, its most likely trigger was OSA associated hypoxaemia. Other pathogenetic mechanisms include intrathoracic pressure swings and sympathetic activation. The fact that the AF stopped after a phase of undisturbed breathing also suggests that the heart rhythm disorder was induced by OSA. We presume that the absence of these stimuli led to atrial electrical stabilisation and the return to sinus rhythm.

R Schulz, H J Eisele, W Seeger

Department of Pulmonary and Critical Care Medicine, Justus-LiebigUniversity, Giessen, Germany Correspondence to: Dr R Schulz, Department of Pulmonary and Critical Care Medicine, Justus-Liebig-University, Klinikstrasse 36, 35392 Giessen, Germany; Richard.Schulz@innere.med.uni-giessen.de

Conflicts of interest: none.

\section{REFERENCES}

1 Kanagala R, Murali NS, Friedman PA, et al. Obstructive sleep apnea and the recurrence of atrial fibrillation. Circulation 2003;107:2589-94.

2 Gami AS, Pressman G, Caples SM, et al. Association of atrial fibrillation and obstructive sleep apnea. Circulation 2004;110:364-7. 